\title{
Émile DURKHEIM (1858-1917)
}

SOCIOLOGUE FRANÇAIS

(1894)

\section{"Le crime \\ phénomène normal"}

Un document produit en version numérique par Jean-Marie Tremblay, bénévole, professeur de sociologie au Cégep de Chicoutimi

Courriel: jean-marie tremblay@uqac.ca

Site web pédagogique : http://www.uqac.ca/jmt-sociologue/

Dans le cadre de la collection: "Les classiques des sciences sociales"

Site web: http://classiques.uqac.ca/

Une collection développée en collaboration avec la Bibliothèque

Paul-Émile-Boulet de l'Université du Québec à Chicoutimi

Site web: http://bibliotheque.uqac.ca/ 
Cette édition électronique a été réalisée par Jean-Marie Tremblay, bénévole, professeur de sociologie au Cégep de Chicoutimi

à partir de l'article de :

Émile Durkheim, "Le crime, phénomène normal”. Un article publié dans Déviance et criminalité. Textes réunis par Denis Szabo avec la collaboration d'André Normandeau, pp. 76-82. Paris: Librairie Armand Colin, 1970, 378 pp. Collection U2.

Source: Les règles de la méthode sociologique (1894), Paris, P.U.F., 14e édition, 1960, pp. 65-72.

Polices de caractères utilisée :

Pour le texte: Times New Roman, 14 points.

Pour les citations: Times New Roman 12 points.

Pour les notes de bas de page : Times New Roman, 12 points.

Édition électronique réalisée avec le traitement de textes Microsoft Word 2008 pour Macintosh.

Mise en page sur papier format : LETTRE US, 8.5' $x 11^{\prime \prime}$.

Édition numérique réalisée le 14 mai 2006, [revue et corrigée par Monsieur Régis Roussillon, professeur de Sciences Economiques et Sociales en France le 3 mai 2013]. Chicoutimi, Ville de Saguenay, Québec.

Courriel : Régis Roussillon : regis.roussillon@laposte.net. 


\section{Émile Durkheim}

\section{“Le crime, phénomène normal” (1894)}

\section{COLLECTION U,

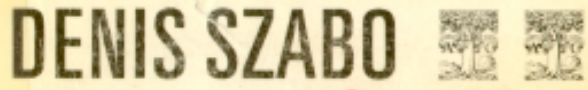

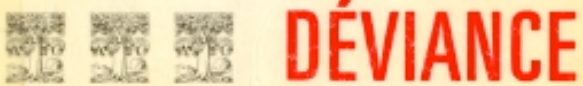 ET CRIMINALITÉ 楚 TEXTES \\ ARMAND COLIN}

Un article publié dans Déviance et criminalité. Textes réunis par Denis Szabo avec la collaboration d'André Normandeau, pp. 76-82. Paris: Librairie Armand Colin, 1970, 378 pp. Collection U2.

Source: Les règles de la méthode sociologique (1894), Paris, P.U.F., 14e édition, 1960, pp. 65-72. 
Émile Durkheim,

"Le crime, phénomène normal". (1894)

Un article publié dans Déviance et criminalité. Textes réunis par Denis Szabo avec la collaboration d'André Normandeau, pp. 76-82. Paris: Librairie Armand Colin, 1970, 378 pp. Collection U2.

Source: Les règles de la méthode sociologique (1894), Paris, P.U.F., 14e édi-tion, 1960, pp. 65-72.

Le crime est normal, parce qu'une société qui en serait exempte est tout à fait impossible; telle est la première évidence paradoxale que fait surgir la réflexion sociologique.

S'il est un fait dont le caractère pathologique parait incontestable, c'est le crime. Tous les criminologistes s'entendent sur ce point. S'ils expliquent cette morbidité de manières différentes, ils sont unanimes à la reconnaître. Le problème, cependant, demandait à être traité avec moins de promptitude.

Appliquons, en effet, les règles précédentes. Le crime ne s'observe pas seulement dans la plupart des sociétés de telle ou telle espèce, mais dans toutes les sociétés de tous les types. Il n'en est pas où il n'existe une criminalité. Elle change de forme, les actes qui sont ainsi qualifiés ne sont pas partout les mêmes ; mais, partout et toujours, il y a eu des hommes qui se conduisaient de manière à attirer sur eux la répression pénale. $\mathrm{Si}$, du moins, à mesure que les sociétés passent des types inférieurs aux plus élevés, le taux de la criminalité, c'est-à-dire le rapport entre le chiffre annuel des crimes et celui de la population, tendait à baisser, on pourrait croire que, tout en restant un phénomène 
normal, le crime, cependant, tend à perdre ce caractère. Mais nous n'avons aucune raison qui nous permette de croire à la réalité de cette régression. Bien des faits sembleraient plutôt démontrer l'existence d'un mouvement en sens inverse. Depuis le commencement du siècle, la statistique nous fournit le moyen de suivre la marche de la criminalité ; or, elle a partout augmenté. En France, l'augmentation est près de $300 \%$. Il n'est donc pas de phénomène qui présente de la manière la plus irrécusée tous les symptômes de la normalité, puisqu'il apparaît comme étroitement lié aux conditions de toute vie collective. Faire du crime une maladie sociale, ce serait admettre que la maladie n'est pas quelque chose d'accidentel, mais, au contraire, dérive, dans certains cas, de la constitution fondamentale de l'être vivant; ce serait effacer toute distinction entre le physiologique et le pathologique. Sans doute, il peut se faire que le crime lui-même ait des formes anormales; c'est ce qui arrive quand, par exemple, il atteint un taux exagéré. Il n'est pas douteux, en effet, que cet excès ne soit de nature morbide. Ce qui est normal, c'est simplement qu'il y ait une criminalité, pourvu que celleci atteigne et ne dépasse pas, pour chaque type social, un certain niveau qu'il n'est peut-être pas impossible de fixer conformément aux règles précédentes ${ }^{1}$.

Nous voilà en présence d'une conclusion, en apparence assez paradoxale. Car il ne faut pas s'y méprendre. Classer le crime parmi les phénomènes de sociologie normale, ce n'est pas seulement dire qu'il est un phénomène inévitable quoique regrettable, dû à l'incorrigible méchanceté des hommes; c'est affirmer qu'il est un facteur de la santé publique, une partie intégrante de toute société saine. Ce résultat est, au premier abord, assez surprenant pour qu'il nous ait nous-même déconcerté et pendant longtemps. Cependant, une fois que l'on a dominé cette première impression de surprise, il n'est pas difficile de trouver les raisons qui expliquent cette normalité, et, du même coup, la confirment.

De ce que le crime est un phénomène de sociologie normale, il ne suit pas que le criminel soit un individu normalement constitué au point de vue biologique et psychologique. 'Les deux questions sont indépendantes l'une de l'autre. On comprendra mieux cette indépendance, quand nous aurons montré plus loin la différence qu'il y a entre les faits psychiques et les faits sociologiques. 
En premier lieu, le crime est normal parce qu'une société qui en serait exempte est tout à fait impossible.

Le crime, nous l'avons montré ailleurs, consiste dans un acte qui offense certains sentiments collectifs, doués d'une énergie et d'une netteté particulières. Pour que, dans une société donnée, les actes réputés criminels pussent cesser d'être commis, il faudrait donc que les sentiments qu'ils blessent se retrouvassent dans toutes les consciences individuelles sans exception et avec le degré de force nécessaire pour contenir les sentiments contraires. Or, à supposer que cette condition pût être effectivement réalisée, le crime ne disparaîtrait pas pour cela, il changerait seulement de forme ; car la cause même qui tarirait ainsi les sources de la criminalité en ouvrirait immédiatement de nouvelles.

En effet, pour que les sentiments collectifs que protège le droit pénal d'un peuple, à un moment déterminé de son histoire, parviennent ainsi à pénétrer dans les consciences qui leur étaient jusqu'alors fermées ou à prendre plus d'empire là où ils n'en avaient pas assez, il faut qu'ils acquièrent une intensité supérieure à celle qu'ils avaient jusqu'alors. Il faut que la communauté dans son ensemble les ressente avec plus de vivacité ; car ils ne peuvent pas puiser à une autre source la force plus grande qui leur permet de s'imposer aux individus qui, naguère, leur étaient les plus réfractaires. Pour que les meurtriers disparaissent, il faut que l'horreur du sang versé devienne plus grande dans ces couches sociales où se recrutent les meurtriers; mais, pour cela, il faut qu'elle devienne plus grande dans toute l'étendue de la société. D'ailleurs, l'absence même du crime contribuerait directement à produire ce résultat; car un sentiment apparaît comme beaucoup plus respectable quand il est toujours et uniformément respecté.

Mais on ne fait pas attention que ces états forts de la conscience commune ne peuvent être ainsi renforcés sans que les états plus faibles, dont la violation ne donnait précédemment naissance qu'à des fautes purement morales, ne soient renforcées du même coup ; car les seconds ne sont que le prolongement, la forme atténuée des premiers. Ainsi, le vol et la simple indélicatesse ne froissent qu'un seul et même sentiment altruiste, le respect de la propriété d'autrui. Seulement ce même sentiment est offensé plus faiblement par l'un de ces actes que par l'autre ; et comme, d'autre part, il n'a pas dans la moyenne des 
consciences une intensité suffisante pour ressentir vivement la plus légère de ces deux offenses, celle-ci est l'objet d'une plus grande tolérance. Voilà pourquoi on blâme simplement l'indélicat tandis que le voleur est puni. Mais si ce même sentiment devient plus fort, au point de faire taire dans toutes les consciences le penchant qui incline l'homme au vol, il deviendra plus sensible aux lésions qui, jusqu'alors, ne le touchaient que légèrement; il réagira donc contre elles avec plus de vivacité ; elles seront l'objet d'une réprobation plus énergique qui fera passer certaines d'entre elles, de simples fautes morales qu'elles étaient, à l'état de crimes. Par exemple, les contrats indélicats ou indélicatement exécutés, qui n'entraînent qu'un blâme public ou des réparations civiles, deviendront des délits. Imaginez une société de saints, un cloître exemplaire et parfait. Les crimes proprement dits y seront inconnus ; mais les fautes qui paraissent vénielles au vulgaire y soulèveront le même scandale que fait le délit ordinaire auprès des consciences ordinaires. Si donc cette société se trouve armée du pouvoir de juger et de punir, elle qualifiera ces actes de criminels et les traitera comme tels. C'est pour la même raison que le parfait honnête homme juge ses moindres défaillances morales avec une sévérité que la foule réserve aux actes vraiment délictueux. Autrefois, les violences contre les personnes étaient plus fréquentes qu'aujourd'hui parce que le respect pour la dignité individuelle était plus faible. Comme il s'est accru, ces crimes sont devenus plus rares ; mais aussi, bien des actes qui lésaient ce sentiment sont entrés dans le droit pénal dont ils ne relevaient primitivement pas ${ }^{2}$.

On se demandera peut-être, pour épuiser toutes les hypothèses logiquement possibles, pourquoi cette unanimité ne s'étendrait pas à tous les sentiments collectifs sans exception; pourquoi même les plus faibles ne prendraient pas assez d'énergie pour prévenir toute dissidence. La conscience morale de la société se retrouverait tout entière chez tous les individus et avec une vitalité suffisante pour empêcher tout acte qui l'offense, les fautes purement morales aussi bien que les crimes. Mais une uniformité aussi universelle et aussi absolue est radicalement impossible ; car le milieu physique immédiat dans lequel chacun de nous est placé, les antécédents héréditaires, les influences sociales dont nous dépendons varient d'un individu à l'autre et, par

2 Calomnies, injures, diffamation, vol, etc. 
suite, diversifient les consciences. Il n'est pas possible que tout le monde se ressemble à ce point, par cela seul que chacun a son organisme propre et que ces organismes occupent des portions différentes de l'espace. C'est pourquoi, même chez les peuples inférieurs, où l'originalité individuelle est très peu développée, elle n'est cependant pas nulle. Ainsi donc, puisqu'il ne peut pas y avoir de société où les individus ne divergent plus ou moins du type collectif, il est inévitable aussi que, parmi ces divergences, il y en ait qui présentent un caractère criminel. Car ce qui leur confère ce caractère, ce n'est pas leur importance intrinsèque, mais celle que leur prête la conscience commune. Si donc celle-ci est plus forte, si elle a assez d'autorité pour rendre ces divergences très faibles en valeur absolue, elle sera aussi plus sensible, plus exigeante, et, réagissant contre de moindres écarts avec l'énergie qu'elle ne déploie ailleurs que contre des dissidences plus considérables, elle leur attribue la même gravité, c'est-à-dire qu'elle les marquera comme criminels.

Le crime est donc nécessaire : il est lié aux conditions fondamentales de toute vie sociale, mais, par cela même, il est utile; car ces conditions dont il est solidaire sont elles-mêmes indispensables à l'évolution normale de la morale et du droit.

En effet, il n'est plus possible aujourd'hui de contester que non seulement le droit et la morale varient d'un type social à l'autre, mais encore qu'ils changent pour un même type si les conditions de l'existence collective se modifient. Mais, pour que ces transformations soient possibles, il faut que les sentiments collectifs qui sont à la base de la morale ne soient pas réfractaires au changement, par conséquent, n'aient qu'une énergie modérée. S'ils étaient trop forts, ils ne seraient plus plastiques. Tout arrangement, en effet, est un obstacle au réarrangement, et cela d'autant plus que l'arrangement primitif est plus solide. Plus une structure est fortement accusée, plus elle oppose de résistance à toute modification et il en est des arrangements fonctionnels comme des arrangements anatomiques. Or, s'il n'y avait pas de crimes, cette condition ne serait pas remplie ; car une telle hypothèse suppose que les sentiments collectifs seraient parvenus à un degré d'intensité sans exemple dans l'histoire. Rien n'est bon indéfiniment et sans mesure. Il faut que l'autorité dont jouit la conscience morale ne soit pas excessive ; autrement, nul n'oserait y porter la main et elle se figerait 
trop facilement sous une forme immuable. Pour qu'elle puisse évoluer, il faut que l'originalité puisse se faire jour ; or pour que celle de l'idéaliste qui rêve de dépasser son siècle puisse se manifester, il faut que celle du criminel, qui est au-dessous de son temps, soit possible. L'une ne va pas sans l'autre.

Ce n'est pas tout. Outre cette utilité indirecte, il arrive que le crime joue lui-même un rôle utile dans cette évolution. Non seulement il implique que la voie reste ouverte aux changements nécessaires, mais encore, dans certains cas, il prépare directement ces changements. Non seulement, là où il existe, les sentiments collectifs sont dans l'état de malléabilité nécessaire pour prendre une forme nouvelle, mais encore il contribue parfois à prédéterminer la forme qu'ils prendront. Que de fois, en effet, il n'est qu'une anticipation de la morale à venir, un acheminement vers ce qui sera! D'après le droit athénien, Socrate était un criminel et sa condamnation n'avait rien que de juste. Cependant son crime, à savoir l'indépendance de sa pensée, était utile à préparer une morale et une foi nouvelles dont les Athéniens avaient alors besoin parce que les traditions dont ils avaient vécu jusqu'alors n'étaient plus en harmonie avec leurs conditions d'existence. Or le cas de Socrate n'est pas isolé ; il se reproduit périodiquement dans l'histoire. La liberté de penser dont nous jouissons actuellement n'aurait jamais pu être proclamée si les règles qui la prohibaient n'avaient été violées avant d'être solennellement abrogées. Cependant, à ce moment, cette violation était un crime, dans la généralité des consciences. Et néanmoins ce crime était utile puisqu'il préludait à des transformations qui, de jour en jour, devenaient plus nécessaires. La libre philosophie a eu pour précurseurs les hérétiques de toute sorte que le bras séculier a justement frappés pendant tout le cours du Moyen Âge et jusqu'à la veille des temps contemporains.

De ce point de vue, les faits fondamentaux de la criminologie se présentent à nous sous un aspect entièrement nouveau. Contrairement aux idées courantes, le criminel n'apparaît plus comme un être radicalement insociable, comme une sorte d'élément parasite, de corps étranger et inassimilable, introduit au sein de la société ${ }^{3}$; c'est un

Nous avons nous-même commis l'erreur de parler ainsi du criminel, faute d'avoir appliqué notre règle (Division du travail social, p. 395, 396). 
agent régulier de la vie sociale. Le crime, de son côté, ne doit plus être conçu comme un mal qui ne saurait être contenu dans de trop étroites limites ; mais, bien loin qu'il y ait lieu de se féliciter quand il lui arrive de descendre trop sensiblement au-dessous du niveau ordinaire, on peut être certain que ce progrès apparent est à la fois contemporain et solidaire de quelque perturbation sociale. C'est ainsi que jamais le chiffre des coups et blessures ne tombe aussi bas qu'en temps de disette ${ }^{4}$. En même temps et par contrecoup, la théorie de la peine se retrouve renouvelée ou, plutôt, à renouveler. $\mathrm{Si}$, en effet, le crime est une maladie, la peine en est le remède et ne peut être conçue autrement, aussi toutes les discussions qu'elle soulève portent-elles sur le point de savoir ce qu'elle doit être pour remplir son rôle de remède. Mais si le crime n'a rien de morbide, la peine ne saurait avoir pour objet de le guérir et sa vraie fonction doit être cherchée ailleurs.

Fin du texte

4 D'ailleurs, de ce que le crime est un fait de sociologie normale, il ne suit pas qu'il ne faille pas le hair. La douleur, elle non plus, n'a rien de désirable, l'individu la hait comme la société hait le crime, et pourtant elle relève de la physiologie normale. Non seulement elle dérive nécessairement de la constitution même de tout être vivant, mais elle joue un rôle utile dans la vie et pour lequel elle ne peut être remplacée. Ce serait donc dénaturer singulièrement notre pensée que de la présenter comme une apologie du crime. Nous ne songerions même pas à protester contre une telle interprétation, si nous ne savions à quelles étranges accusations on s'expose et à quels malentendus, quand on entreprend d'étudier les faits moraux objectivement et d'en parler dans une langue qui n'est pas celle du vulgaire. 